\title{
Caracterização do processo de rigor mortis dos músculos da paleta (Triceps brachii) e dos músculos duros (Extensor/ Flexor) de javali (Sus scrofa) e maciez da carne
}

\section{Characterization of rigor mortis process of picnic shoulders muscles (Triceps brachii) and of forefoot muscles (Extensor/ Flexor) of wild boar (Sus scrofa) and meat tenderness}

Alfredo Tavares Fernandez, ${ }^{*}$ Teófilo José Pimentel da Silva, ${ }^{* *}$ Mônica Queiroz de Freitas, ${ }^{* \star}$ Juliana Alves de Carvalho***

\begin{abstract}
Resumo
O objetivo do presente trabalho foi caracterizar o desenvolvimento do processo de rigor mortis dos músculos Triceps brachii e Extensor/Flexor, de seis carcaças de javalis, criados semiextensivos, durante o pré-resfriamento industrial e a maciez da carne, utilizando medidas de temperatura de carcaças e da câmara de resfriamento, de $\mathrm{pH}$, de comprimento de sarcômero e da força de cisalhamento, nos intervalos de tempo de 0,$5 ; 3,0 ; 5,0 ; 7,0 ; 9,0 ; 12,0$ e 24,0 horas após a sangria. A temperatura da câmara fria variou de $19,6^{\circ} \mathrm{C}(0,5 \mathrm{~h})$ a $-0,5^{\circ} \mathrm{C}(24 \mathrm{~h})$ e a temperatura média das carcaças foi de $39,07^{\circ} \mathrm{C}$ e $0,28^{\circ} \mathrm{C}$, respectivamente. $\mathrm{O}$ pH médio inicial do músculo Triceps brachii foi de 6,64 e o final 5,52 e nos músculos Extensor/Flexor foi de $6,79(0,5 \mathrm{~h})$ e 5,68 (24h), sendo de maior valor nos músculos Extensor/Flexor. A caracterização da contração máxima de sarcômero durante o processo de rigor mortis ocorreu na 7a hora após sangria no músculo Triceps brachii $(1,61 \mu \mathrm{m})$ e na 9a hora nos músculos Extensor/Flexor $(1,63 \mathrm{~mm})$ e o músculo T. brachii apresentou maior tamanho de sarcômero. As perdas por cozimento foram proporcionais à força de cisalhamento onde as amostras de carne da paleta de javali foram consideradas macias, ou seja, inferiores a $5 \mathrm{~kg}$.
\end{abstract}

Palavras-chave: músculos, carcaça de javali, rigor mortis, pH, maciez.

\begin{abstract}
The objective of the present work was to characterize the development of the process of rigor mortis of the Triceps brachii and Extensor/Flexor muscles, of 06 wild boars, created semi-extensive, during the industrial pre-chilling and tenderness of the meat, using measures of temperature of carcasses and of the cold room, of $\mathrm{pH}$, of sarcomere length and of the shear force, in the intervals of time of $0.5 ; 3.0 ; 5.0 ; 7.0 ; 9.0 ; 12.0$ and 24.0 hours after exsanguination. The temperature of the cold room changed from $19.6^{\circ} \mathrm{C}(0.5 \mathrm{~h})$ to $-0.5^{\circ} \mathrm{C}(24 \mathrm{~h})$ and the mean temperature of the carcasses was from $39.07^{\circ} \mathrm{C}$ to $0.28^{\circ} \mathrm{C}$, respectively. The mean initial pH of the muscle Triceps brachii was of 6.64 and final of 5.52 and Extensor/Flexor muscles was $6.79(0.5 \mathrm{~h})$ and $5.68(24 \mathrm{~h})$, being a bigger value in the muscles. Extensor/Flexor. The characterization of maximum contraction of the sarcomere during the of rigor mortis process took place in the 7th hour after exsanguination in the muscle Triceps brachii and in the 9th hour in the muscles Extensor/Flexor and the muscle Triceps brachii presented bigger sarcomere lenght. The cooking loss was proportional to the shear force where the samples of meat of wild boar shoulder were considered tender, in other words, inferior to $5 \mathrm{~kg}$.
\end{abstract}

Keywords: muscles, wild boar carcass, rigor mortis, $\mathrm{pH}$, tenderness.

\section{Introdução}

As correntes conservacionistas atuais apontam a criação comercial de animais silvestres como uma forma de preservação de espécies que podem ser exploradas como fonte de proteína para alimentação (Lavorenti e Nogueira Filho, 1995).

A carne de javali apresentou boa aceitabilidade em análises sensoriais sendo considerada saudável em relação ao colesterol e nível calórico de acordo com as exigências da "American Heart Association" (Lima, 2002).

Nos animais abatidos, com a sangria, se interrompe a oxigenação dos músculos paralisando a respiração celular e ocasionando a queda do potencial de óxido-redução dando início à glicólise anaeróbica que, junto com a fosfocreatina, tentam produzir ATP mas este esgota-se rapidamente, levando a inextensibilidade dos músculos (Pardi et al., 2001).

* Programa de Pós-Graduação em Medicina Veterinária (Doutorado) - Higiene Veterinária e Processamento Tecnológico de Produtos de Origem Animal, Faculdade de Veterinária, Universidade Federal Fluminense. E-mail: altafe@ig.com.br

** Departamento de Tecnologia dos Alimentos da Faculdade de Veterinária, Universidade Federal Fluminense.

*** Aluna de iniciação científica.

A quem enviar correspondência. E-mail: altafe@ig.com.br 
A queda do $\mathrm{pH}$, pelo acúmulo de ácido lático, constitui um dos fatores mais marcantes na transformação do músculo em carne sendo decisiva na qualidade da carne. No caso de suínos, este valor de 6,68 a 6,72 cai para 5,6 e 5,7 entre seis a oito horas após sangria até alcançar valores entre 5,3 e 5,7 em 24 horas após o abate. Em músculos com fibras brancas ou de contração rápida, o pH final atinge valores menores enquanto nos músculos de contração lenta (fibras vermelhas), o pH final fica mais elevado. São também variáveis a temperatura que ocorre o processo post mortem e fatores de estresse que influenciam nas características da carne como comprimento de sarcômero, maciez, coloração, suculência e rendimento industrial (Jeremiah et al., 1991; Feldhusen e Kuhne, 1997; Heneckel et al., 1997; Wal et al., 1997; Pinheiro et al., 2001; Marchiori e Felício, 2003; Zochowska et al., 2005; Mabry et al., 2006).

As características do processo de rigor mortis de carcaças de javalis ainda não foram estabelecidas nas condições brasileiras e na literatura pesquisada, como já foram observadas em suínos (Pinheiro et al., 2001), em perus (Santos et al., 2006) e em avestruzes (Costa et al., 2006).

Os objetivos desta pesquisa foram: caracterizar o comportamento do processo de rigor mortis de carcaças de javalis durante o resfriamento industrial mensurando peso, temperatura da carcaça e da câmara frigorífica, $\mathrm{pH}$ e tamanho de sarcômero nos músculos Triceps brachii e Extensor/Flexor e análise de maciez (perdas por cocção e força de cisalhamento).

\section{Material e métodos}

As amostras foram constituídas de carcaças de javalis utilizando músculos da paleta (Triceps brachii) e músculos duros (Extensor/Flexor) os quais foram obtidos de seis animais machos com aproximadamente 165 dias de vida, com peso vivo entre 45 e $53 \mathrm{~kg}$, criados semiextensivos, nas mesmas condições e escolhidos aleatoriamente no matadouro frigorífico Marchiori (SIF-1504) - Amparo-SP. Os animais foram insensibilizados por atordoamento elétrico conforme o Regulamento Técnico de Métodos de Insensibilização para o Abate Humanitário de Animais de Açougue (Brasil, 2000) e segundo as normas técnicas, higiênicas e sanitárias legais (Brasil, 1997).

As meias carcaças foram conduzidas devidamente identificadas para a câmara de resfriamento $\left(19,6^{\circ} \mathrm{C}\right.$ a temperatura média e $82,2 \%$ a umidade relativa), na qual foi realizada a tomada de temperatura com um termo-higrômetro, nos intervalos de tempo de 0,5;3,0; 5,0; 7,0; 9,0; 12,0 e $24,0 \mathrm{~h}$ após sangria. Nos mesmos intervalos foi tomada a temperatura das meias carcaças introduzindo-se a haste metálica de um termômetro digital na profundidade de $3 \mathrm{~cm}$ da massa muscular do pernil. Amostras de cinco grama dos músculos Triceps brachii e Extensor/Flexor foram obtidas nos respectivos intervalos de tempo para medição do $\mathrm{pH}$ com potenciômetro Handylab I-Schott, utilizando uma solução homogeneizada com $10 \mathrm{~g}$ da amostra em $100 \mathrm{~mL}$ de água, conforme Silva et al. (1992). Paralelamente, com auxílio de pinça, bisturi e garra metálica dupla (para evitar contração ou distensão das fibras) foram removidas amostras (em duplicata) dos músculos Triceps brachii e Extensor/Flexor com comprimento de $15 \mathrm{~mm}$, largura de $10 \mathrm{~mm}$ e espessura de $5 \mathrm{~mm}$, previamente fixadas pelas garras identificadas pelo número de carcaça, nome do músculo e intervalo de tempo após sangria. Em ato contínuo, essas amostras foram colocadas em frascos plásticos de boca larga contendo formalina tamponada 10\% (200 mL). Após a fixação, as amostras foram clivadas, desidratadas, clarificadas, incluídas em parafina e seccionadas em um micrômetro (Pika-Seiko) com espessura de cinco micra. Os cortes histológicos foram corados com Hematoxilina Fosfotúngstica de Mallory segundo a técnica recomendada por Behmer et al. (1976). As lâminas foram lidas utilizando um microscópio óptico Nikon com luz visível em objetiva de imersão segundo Sloss e Kemp (1978). Este método é baseado na contagem de 10 sarcômeros de seis miofibrilas diferentes, com a medição através de uma régua milimetrada dentro de uma ocular com escala de 10 micrômetros. A média obtida foi multiplicada pelo fator de correção da objetiva de imersão $(0,8 \mu \mathrm{m})$ e o valor expresso em micrômetros $(\mu \mathrm{m})$.

Para a análise de perdas de suco por cozimento e da força de cisalhamento, as amostras de paleta (Triceps brachii) com peso de 62,5 a $79,5 \mathrm{~g}$ foram cozidas até a temperatura interna de $70^{\circ} \mathrm{C}$ e mantidas a temperatura ambiente como recomendam Morgan et al. (1991). As amostras foram pesadas e a seguir, realizados os cálculos para determinação do percentual de perdas de suco no processo de cozimento (peso antes do cozimento - peso após o cozimento dividido pelo peso antes do cozimento x100) segundo fórmula de Diles et al. (1994).

Para o teste de maciez, foram retirados seis cilindros de carne cozida resfriada de $1,27 \mathrm{~cm}$ de diâmetro de espessura no sentido longitudinal das fibras musculares de cada amostra (Koohmaraiae et al., 1990) e cisalhados ao meio na máquina "Warner-Blatzer Meat Shear Force 3000". (Kerth et al., 1995) para obtenção dos valores de força de cisalhamento $(\mathrm{kg})$.

Para a comparação dos valores obtidos utilizou-se a análise de variância (delineamento inteiramente casualizado em fatorial $2^{7}$ ); o teste de Tukey com significância de 5\%, empregando o programa SASß 8.2 (SAS, 2001).

\section{Resultados e discussão}

De acordo com a Figura 1, os valores médios de temperatura das carcaças de javalis foram de $39,07^{\circ} \mathrm{C}(0,5 \mathrm{~h}), 12,0^{\circ} \mathrm{C}(7 \mathrm{~h})$ e $0,28^{\circ} \mathrm{C}(24 \mathrm{~h})$; enquanto os valores de temperatura da câmara de resfriamento foram $19,6^{\circ} \mathrm{C}(0,5 \mathrm{~h}), 7,2^{\circ} \mathrm{C}(7 \mathrm{~h})$ e $0,5^{\circ} \mathrm{C}(24 \mathrm{~h})$. A análise estatística não revelou diferença significativa $(p>0,05)$ quanto aos valores de temperatura de carcaças no intervalo de tempo de 7 e 9 horas.

Esses resultados obtidos estão em concordância com os dados observados por Marchiori e Felício (2003) que encontraram valores de temperatura do músculo Longissimus dorsi de javali de $30,2^{\circ} \mathrm{C}(1 \mathrm{~h}), 12,4^{\circ} \mathrm{C}(6 \mathrm{~h})$ e $5,4^{\circ} \mathrm{C}(24 \mathrm{~h}) . \mathrm{Na}$ última medição de temperatura (24h) houve diferença de mais de cinco graus centígrados no trabalho de Marchiori e Felício (2003) da obtida neste experimento similar, provavelmente devido a fatores intrínsecos (características de carcaças como peso e espessura de gordura subcutânea) e fatores extrínsecos (câmara fria: lotação, espaçamento entre carcaças, frio produzido, ventilação etc.) podem estar relacionados com esta diferença. No entanto, os valores médios de temperatura de carcaça e de câmara de resfriamento obtidos ocorreram dentro dos padrões estabelecidos para evitar o rigor de resfriamento ou 


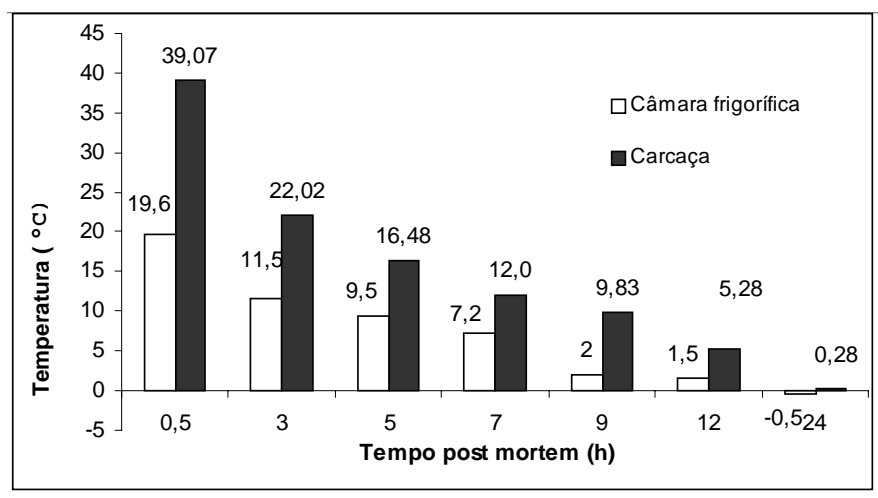

Figura 1: Variação da temperatura $\left({ }^{\circ} \mathrm{C}\right)$ da câmara frigorífica e dos valores médios de temperatura das seis carcaças de javalis nos diferentes intervalos de tempo

encurtamento pelo frio. A queda de temperatura é fundamental para remoção do calor latente da carcaça o qual poderia facilitar o crescimento microbiano em função da perda da homeostasia pela morte do animal. Entretanto, essa queda de temperatura necessita ser monitorada para evitar o encurtamento pelo frio, - bloqueio da resolução do processo de rigor mortis que resultaria em carne dura (Pardi et al., 2001).

Os valores médios encontrados na determinação de $\mathrm{pH}$ nos músculos Triceps brachii e Extensor/Flexor (Figura 2) após sangria e durante o resfriamento industrial, foram de 6,64 e 6,79 aos 30 minutos, de 5,87 e 6,13 na $7^{\text {a. }}$. hora e 5,62 e 5,68 na $24^{\text {a }}$. hora, respectivamente. As comparações feitas pelo teste de Tukey revelaram existir diferença significativa $(p<0,05)$ dos valores médios de $\mathrm{pH}$ entre esses músculos em todos os intervalos de tempo. Isto pode ser explicado pelos músculos Extensor/Flexor possuírem menor conteúdo de glicogênio devido à maior atividade física in vivo, pela sua maior riqueza em fibras vermelhas e maior atividade oxidativa. Não houve diferença significativa $(p>0,05)$ entre os intervalos de tempo de $7 \mathrm{~h}$ e $9 \mathrm{~h}$ para o músculo Triceps brachii e entre 9h e 12h para os músculos Extensor/Flexor, caracterizando intervalos diferentes do processo de contração máxima do rigor mortis.
Marchiori e Felício (2003) estudaram as mudanças de $\mathrm{pH}$ do músculo Longissimus dorsi de javali durante o período de post mortem e os resultados médios foram de 6,18 (1h), 5,75 (6h) e 5,57 (24h), valores ligeiramente menores que os obtidos neste experimento, provavelmente devido ao músculo Longissimus dorsi possuir mais fibras brancas do que o Triceps brachii. Resultados similares foram obtidos por Heneckel et al. (1997) e Pinheiro et al. (2001) em músculos Longissimus dorsi de suínos que encontraram $\mathrm{pH}$ final de 5,46 e 5,49 , respectivamente, na 24 a hora após sangria, isto em função dos javalis serem criados semiextensivo e não nativo. Por outro lado, Mabry et al. (2006) obtiveram valores de $\mathrm{pH}$ em músculos Longissimus dorsi de suínos, 24 horas após abate superiores, ou seja; de 5,91; 5,85; 5,84; 5,75 e 5,70 nas raças Berkshire, Duroc, Large White, Danbred e Hampshire, respectivamente. Esses valores superiores de $\mathrm{pH}$ podem ter sido em função de uma taxa rápida de préresfriamento, pois a faixa padrão de pH 24 horas após sangria desse músculo no suíno é entre 5,55 e 5,60 (Wall et al., 1997). Não foram encontrados dados na literatura científica consultada sobre pH dos músculos Triceps brachii e Extensor/Flexor de javalis.

Ao estudar as mudanças estruturais nesses músculos durante o processo de rigor mortis encontrou-se valores médios do comprimento de sarcômero do Triceps brachii e

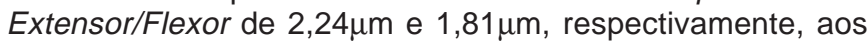
30 minutos ou 0,5 h após sangria (Figura 3 ).

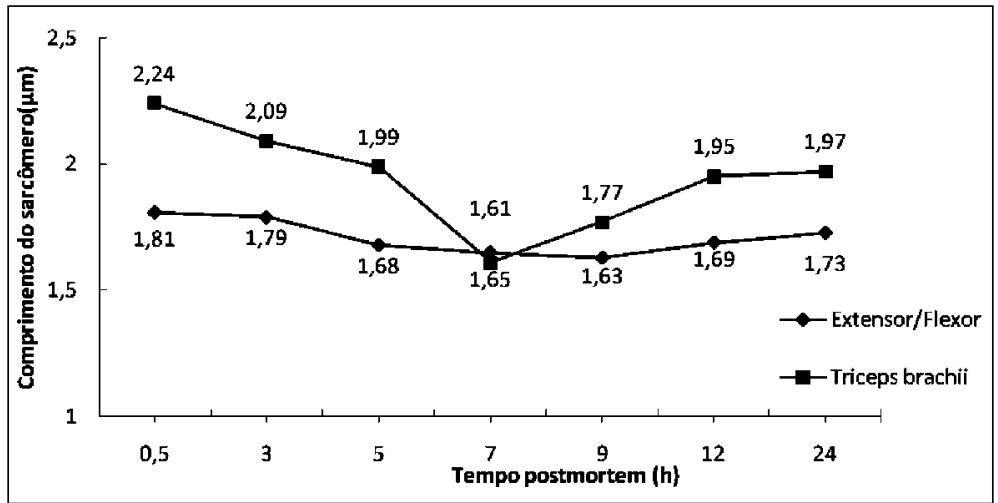

Figura 3: Valores médios $(x \pm s)$ do comprimento de sarcômero $(\mu \mathrm{m})$ dos músculos Triceps brachii - TB e Extensor/Flexor - E/F das carcaças dos seis javalis, nos diferentes tempos $(\mathrm{h})$ após a sangria, durante o resfriamento industrial

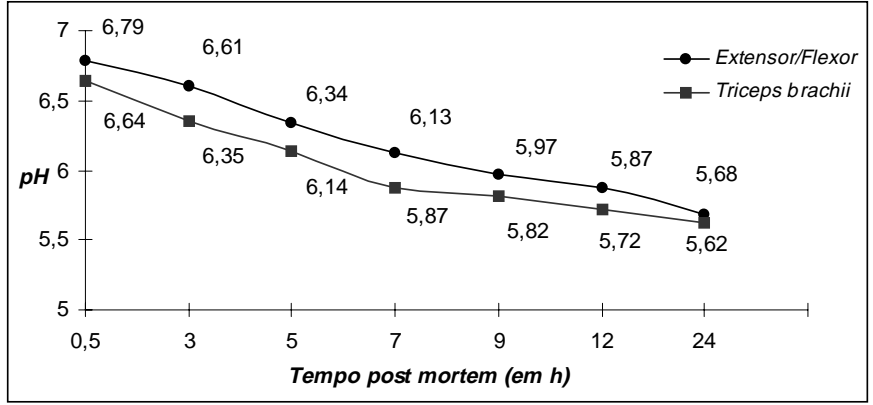

Figura 2: Variação dos valores médios de $\mathrm{pH}$ dos músculos Triceps brachii - TB e Extensor/Flexor - E/F das carcaças de seis javalis nos diferentes intervalos de tempo
Na 7ạ hora o músculo Triceps brachii apresentou seu encurtamento máximo $(1,61 \mu \mathrm{m})$, enquanto o mesmo ocorreu na 9a hora para os músculos Extensor/Flexor (1,63 $\mu \mathrm{m})$. Após a resolução do processo de rigor mortis, ou seja, na 24르 hora o músculo Triceps brachii apresentou maior comprimento de sarcômero $(1,97 \mu \mathrm{m})$ que os Extensor/Flexor $(1,73 \mu \mathrm{m}) .0$ desenvolvimento do processo de rigor mortis dos animais de açougue apresenta diferentes comprimentos dos sarcômeros durante a instalação do processo bioquímico do postmortem. Assim, foram estudadas as mudanças que ocorrem antes e após o rigor mortis e sua influência na maciez do músculo Semispinalis capitis de suínos (Pinheiro et al., 2001) que encontraram comprimento de sarcômero de $1,72 \mu \mathrm{m}$ (1h) e 1,66 $\mathrm{m}$ (24h); do músculo Gastrocnemius de 


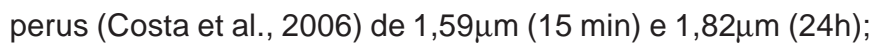
e do músculo Fibularis longus de avestruzes (Santos et al.,

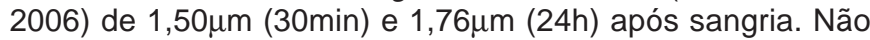
foram encontrados resultados na pesquisa científica referentes a comprimento de sarcômeros em músculos de javali.

Na Figura 4 são mostradas as fotomicrografias das estruturas miofibrilares, o grau de contração máxima do rigor mortis e a resolução após 24 horas da sangria. A caracterização da contração máxima dos sarcômeros do músculo Triceps

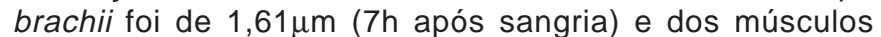
Extensor/Flexor de 1,63 (9h). Observa-se que o músculo

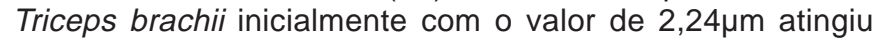
valor mínimo da contração máxima de 1,61 $\mu$ m às sete horas após a sangria e valor de 1,97 $\mu$ m às 24 horas do post mortem ou na resolução do processo de rigor mortis. No que se refere aos músculos Extensor/Flexor, estes apresentaram inicialmente valor médio de $1,81 \mu \mathrm{m}$ chegando ao valor mínimo de 1,63um nove horas após a sangria, e em 24 horas, este valor chegou a $1,73 \mu \mathrm{m}$ na resolução do rigor mortis. Houve diferença significativa $(p<0,05)$ entre os intervalos de tempo para os músculos Extensor/Flexor não havendo diferença significativa entre os demais intervalos de tempo $(p>0,05)$ talvez em função de o sarcômero ser menor, o encolhimento seria também pequeno. Como era de se esperar, também houve diferença significativa $(p<0,05)$ entre os músculos estudados quanto ao comprimento de sarcômeros. Os resultados ocorreram de acordo com o esperado, já que os músculos Extensor/Flexor por apresentarem maior atividade física sendo de tração no animal apresentaria menor comprimento de sarcômero enquanto o músculo Triceps brachii apresentou maior comprimento de sarcômero por ter menor atividade em relação aos músculos Extensor/Flexor. Por outro lado, Heneckel et al. (1997) constataram medidas de 1,76um no sarcômero do músculo Longissimus dorsi de suínos da raça

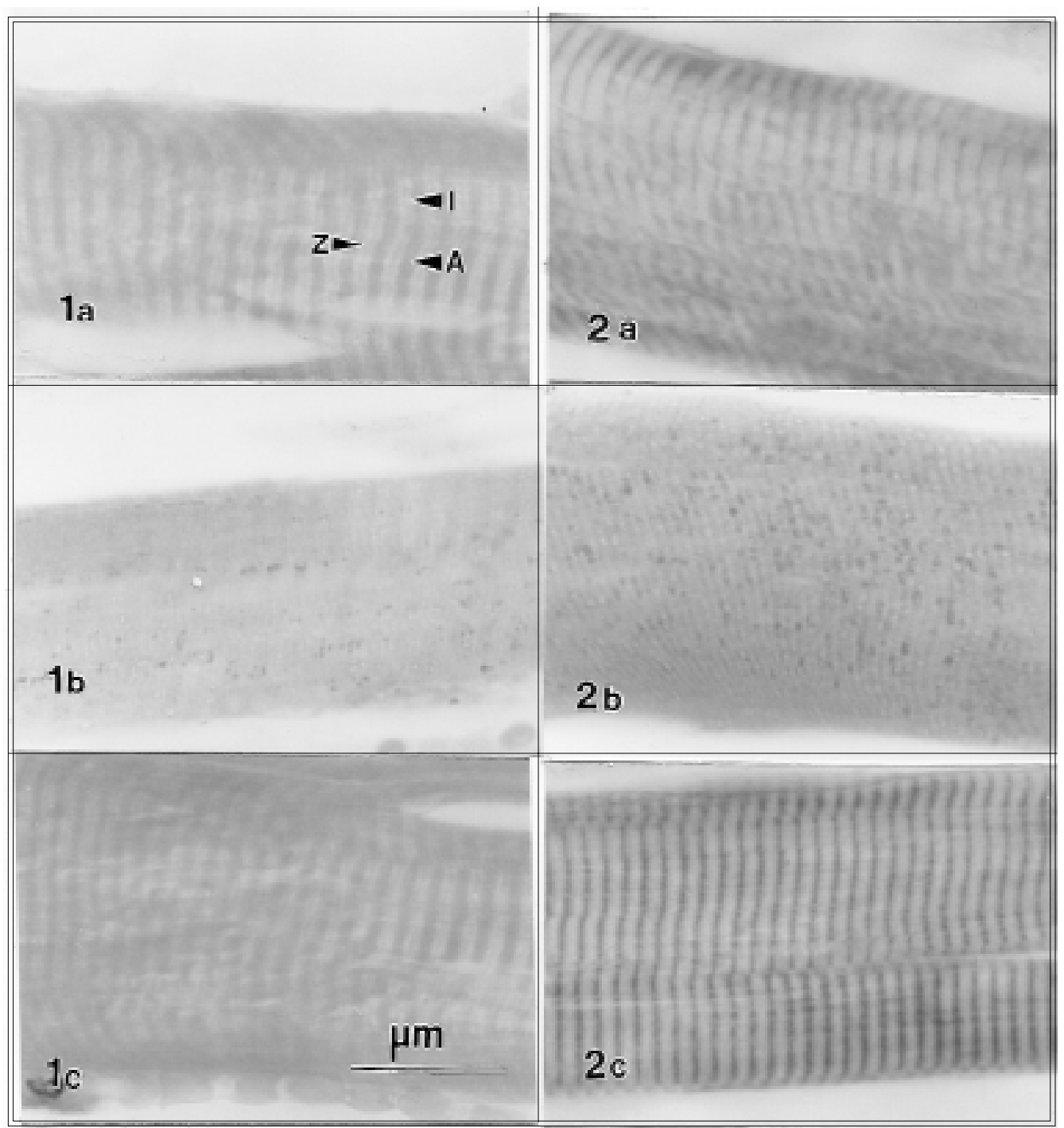

Figura 4: Fotomicrografias dos músculos Triceps brachii e Extensor/Flexor de carcaças de javali durante o resfriamento industrial mostrando as bandas $A$ e I e disco $Z$ (aumento de 1000 vezes), fixado com hematoxilina fosfotúngstica de Mallory, com diferentes tamanhos de sarcômeros: 1 a) músculo Triceps aos 30 minutos após

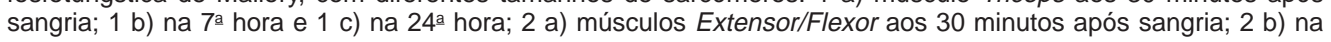
9a hora e na 24a hora 
Landrace após 24 horas de abate. Já Feldhusen e Kühne (1992) trabalhando também com músculos Longissimus dorsi e Semimembranosus de suínos encontraram valores de 1,85 e 1,88um na primeira hora post mortem e após 24

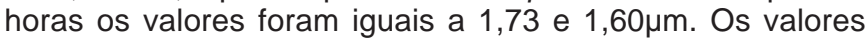
encontrados nos músculos Extensor/Flexor de javali são semelhantes aos valores obtidos pelos autores supracitados e que apesar de serem músculos de tração, os sarcômeros seriam maiores em função do abate em idade menor por ter menor atividade e por outros fatores. Em suínos, a queda do $\mathrm{pH}$ acompanhou gradativamente o desenvolvimento do rigor mortis nos músculos estudados até 24 horas após a sangria, quando. a média inicial das temperaturas das carcaças foi de $39,95^{\circ} \mathrm{C}$ e a final foi de $7^{\circ} \mathrm{C}$. A menor medida do sarcômero do músculo Longissimus dorsi foi obtida na sexta hora após abate $(1,39 \mu \mathrm{m})$ enquanto no músculo Semispinalis capitis, a menor medida foi obtida na oitava hora após abate $(1,37 \mu \mathrm{m})$. Nestes músculos, houve correlação linear entre $\mathrm{pH}$ e comprimento de sarcômero; temperatura e comprimento de sarcômero; pH e temperatura e uma correlação inversa entre tempo e comprimento de sarcômero. Houve correlação inversa entre $\mathrm{pH}$ e tempo de abate; temperatura e tempo de abate (Pinheiro et al., 2001). No presente experimento, a queda da temperatura das carcaças, do $\mathrm{pH}$ e da resolução do processo de post mortem também seguiu gradualmente o desenvolvimento do rigor mortis nos músculos estudados até 24 horas após a sangria.

Com relação às perdas de suco durante o cozimento, as amostras de paleta obtiveram perdas de $28,43 \%$ em 9,4 minutos e a força de cisalhamento foi de 2,06 kg. Não foram encontrados artigos na literatura científica sobre perdas no cozimento de carne de javali mas os resultados foram similares aos de Santos et al. (2006) realizando pesquisa com carne de avestruz e de Costa et al. (2006) com carne de peru os quais verificaram valores entre $24,83 \%$ e $34,20 \%$ e entre $22,33 \%$ e $31,61 \%$, respectivamente. Zochowska et al. (2005) estudaram o efeito do peso de carcaça, ou seja, idade sobre as características da maciez da carne e da estrutura mifibrilar de javalis selvagens abatidos a tiros em uma floresta da Polônia e concluíram que os animais mais jovens resultou uma carne mais macia, menor diâmetro de fibra e alta percentagem de fibras tipo IIB. Fibras tipo IIB têm alta capacidade glicolítica, contração rápida, baixa densidade de mitocondrias, baixa capacidade oxidativa, ou seja, carnes de melhores caracterísicas de maciez e de melhor performance atlética para animais e ser humano(Swatland, 1984). Por outro lado, Jeremiah et al. (1991) consideraram como carnes macias, valores de força de cisalhamento inferiores a $5 \mathrm{~kg}$. No atual experimento, como foram obtidos em todas as amostras valores inferiores a $3 \mathrm{~kg}$ denota-se a significativa maciez das amostras da carne de paleta de javali.

\section{Conclusões}

A queda de temperatura das carcaças de javali e da câmara frigorífica durante o pré-resfriamento industrial ocorreu dentro dos padrões estabelecidos. O declínio de $\mathrm{pH}$ acompanhou o desenvolvimento do processo de rigor mortis em ambos os músculos estudados, sendo de maior valor nos músculos Extensor/Flexor em relação ao Triceps brachii. A caracterização da contração máxima dos sarcômeros durante o

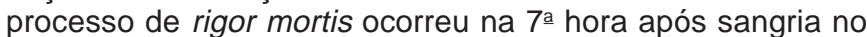
músculo Triceps brachii $(1,61 \mu \mathrm{m})$ e na 9a hora nos músculos Extensor/Flexor $(1,63 \mu \mathrm{m})$ e o músculo $T$. brachii apresentou maior tamanho de sarcômero. As perdas por cozimento foram proporcionais à força de cisalhamento onde as amostras de carne de paleta de javali foram consideradas macias, ou seja, inferiores a $5 \mathrm{~kg}$.

\section{Agradecimentos}

Ao CNPq e ao Matadouro Frigorífico Marchiori Ltda., Amparo, SP.

\section{Referências}

BEHMER, O. A.; JORDENS, J.Z.; GRIFFITS, D. T.; BENEZ, S. M. Manual de técnicas para histologia normal e patológica. São Paulo: EDART - Editora da USP, 1976, 239 p.

BRASIL. Ministério da Agricultura e do Abastecimento. Secretaria Nacional de Defesa Agropecuária. Regulamento de Inspeção Industrial e Sanitária de Produtos de Origem Animal (aprovado pelo Decreto 30.691 de 29/03/1952 alterado pelos decretos nos 1.255 de 25/06/1962, 1.236 de 02/09/1994, no 1.812 de 08/02/1996 e $n^{0}$ 2.244 de 05/06/1997). DIPOA-MAPA, Brasília-DF, 1997, 241p.

Secretaria de Defesa Agropecuária. Regulamento Técnico de Métodos de Insensibilização para o Abate Humanitário de Animais de Açougue. Instrução Normativa no 3 de 17 de janeiro de 2000, (aprovado pela Portaria Ministerial no 574 de 8 de dezembro de 1998, processo ํㅡㄴ 21000,003895/99-17), 2000.

COSTA, F.; SILVA, T. J. P.; FREITAS, M. et al. Caracterização do processo de rigor mortis nos músculos Gastrocnemius e Pectoralis e maciez da carne. Revista Brasileira de Ciência Veterinária, v. 13, p. 165-169, 2006.

DILES, J.J.B.; MILLER, M. F.; OWEN, B. L. Calcium chloride concentration injection time and aging period effects on tenderness, sensory and retail color attributes of loin steaks from mature cows. Journal of Animal Science, v. 72, p. 2017-2021, 1994.
FELDHUSEN, F. ; KÜHNE, M. Effects of ultrarapid chilling and ageing on length of sarcomeres and tenderness of pork. Meat Science, v. 32 , n. 2 , p. 161-171, 1992.

HENECKEL, P.; OKSBJERG, N.; ERLANDSEN, E.; BARTON-GADE, P. BEJERHOLM, C. Histo and biochemical characteristics of the Longissimus dorsimuscle in pigs and their relationship to performance and meat quality. Meat Science, v. 47, n. 3/4, p. 311-321, 1997.

JEREMIAH, L. E.; TONG, A. K. Y.; GIBSON, L. L. The insefulness of muscle color and $\mathrm{pH}$ for segregating beef carcasses into tenderness groups. Meat Science, v. 30, p. 97-114, 1991.

KERTH, C. R.; MILLER, M. F.; RAMSEY, C. B. Improvement of beef tenderness and quality traits with calcium chloride injection in beef loins 48 hours postmortem. Journal of Animal Science, v. 53, n. 6, p. 750-756, 1995.

KOOHMARAIE, M.; WHIPPLE, G.; CROUSE, J. D. Acceleration of postmortem tenderization in lamb and Brahman - cross beef carcasses through infusion of calcium chloride. Journal of Food Science. v. 68, p. 1278-1283, 1990.

LAVORENTI, A.; NOGUEIRA FILHO, S. L. G. Criação do caititu e do queixada em cativeiro. Ciência Hoje, v. 19, n. 114, p. 6-9, 1995.

LIMA, S. C. S. Determinação da composição em ácidos graxos e teor de colesterol por cromatografia gasosa em coluna capilar das carnes de queixada, javali, suína e bovina. 2002. 57p. Dissertação (Mestrado). Instituto de Tecnologia. Universidade Federal Rural do Rio de Janeiro. 
MABRY, J. W.; BAAS, T. J.; MILLER, R. K. The impact of Genetics on Pork quality (Revised) National Pork Board Pork Quality American Meat Association. Disponível em: <http;//www.meatscience.org/pubs/ factsheets/q-genetics49.pdf>. Acesso em: 5 dez. 2006.

MARCHIORI, A. F.; FELICIO, P. E. Qualidade da carne de suíno e de javali comercial. Scientia Agricola, v. 60, n. 1,2003. Disponível em: http://www.scielo.br/scielo.php?script=sci_arttext\&pid=S0103$90162003000100001 \&$ lng=en\&nrm=iso\&tlng=en. Acesso em : 9 set. 2006.

MORGAN, J. B.; MILLER, R. K.; MENDEZ, F. M. et al. Using calcium chloride injection to improve tenderness on beef from mature cows. Journal of Animal Science, v. 69, p. 4469-4476, 1991.

PARDI, M.C., SANTOS, I.F., SOUZA, E.R., PARDI, H.S. Ciência, Higiene e Tecnologia da Carne. Goiânia: CEGRAF - UFG/ Niterói: EdUFF, 2 v., v. 1, 2001, 623 p.

PINHEIRO, R. M. K.; SILVA, T. J. P.; CARVALHO, E. C. Q.; COSTA, F. Temperatura, $\mathrm{pH}$ e comprimento de sarcômero durante o rigor mortis, em carcaça frigorificada de suínos abatidos em São Gonçalo - RJ. Revista Higiene Alimentar, v. 13, n. 82, p. 39-46, 2001.
SANTOS, E. R.; SILVA, T. J. P.; GASPAR, A. et al. Caracterização do processo de rigor mortis em músculos (Gastrocnemius internus e Fibularus longus) de avestruz (Struthio camelus) e maciez da carne. Revista Brasileira de Ciência Veterinária, v. 13, p. 160-164, 2006.

STATISTICAL ANALYSIS SYSTEM - SAS. User's guide. Version 8.2.Cary: 2001, $1686 \mathrm{p}$.

SILVA, T. J. P.; ORCUTT, M. W.; FORREST, J. C.; BRACKER, C. E.; JUDGE, M. Effect of heating rate on shortening, ultrasctructure and fracture behavior of prerigor beef muscle. Meat Science, v. 33, n. 1, p. 1-27, 1992.

SLOSS, M. W. B. S.; KEMP, R. L. A. Veterinary Clinical Parasitology. 5a ed. Ames: lowa State University Press, 1978. 247 p.

SWATLAND, H.J. Structure and development of meat animals. New Jersey, 1984. 436 p.

WALL, P.G. van der; ENGEL, B.; HULSEGGE, B Causes for variation in pork quality. Meat Science, v. 46, n. 4, p. 319-327, 1997.

ZOCHOWSKA, J.; LACHOWICZ, K.; GAJOWIECKI, L. et al. Effects of carcass weight and muscle on texture, structure and myofibre characteristics of wild boar meat. Meat Science, v. 71, n. 2, p. 244 248, 2005. 